\title{
INTEGRAL and RXTE/ASM Observations on IGR J17098-3628
}

\author{
Yu-Peng Chen ${ }^{a}$, Shu Zhang ${ }^{* a}$, Nick Schurch ${ }^{b}$, Jian-Min Wang ${ }^{a, c}$, Werner Collmar ${ }^{d}$, \\ Ti-Pei Li ${ }^{a, e}$, Jin-Lu Qu ${ }^{a}$, Cheng-Min Zhang ${ }^{f}$ \\ ${ }^{a}$ Key Laboratory for Particle Astrophysics, Institute of High Energy Physics, 19B YuQuan Road, \\ Beijing 100049, China \\ ${ }^{b}$ Department of Physics, University of Durham, South Road, Durham DH1 3LE, UK \\ ${ }^{c}$ Theoretical Physics Center for Science Facilities (TPCSF), CAS, China \\ ${ }^{d}$ Max-Planck-Institut für extraterrestrische Physik, P.O. Box 1603, D-85740 Garching, Germany \\ ${ }^{e}$ Center for Astrophysics, Tsinghua University, Beijing 100084, China \\ ${ }^{f}$ National Astronomical Observatories, Chinese Academic of Sciences, Beijing 100012, China \\ E-mail: chenypdihep.ac.cn
}

\begin{abstract}
To probe further the possible nature of the unidentified source IGR J17098-3628, we have carried out a detailed analysis of its long-term time variability as monitored by RXTE/ASM, and of its hard X-ray properties as observed by INTEGRAL. INTEGRAL has monitored this sky region over years and significantly detected IGR J17098-3628 only when the source was in this dubbed active state. In particular, at $\geq 20 \mathrm{keV}$, IBIS/ISGRI caught an outburst in March 2005, lasting for $\sim 5$ days with detection significance of $73 \sigma(20-40 \mathrm{keV})$ and with the emission at up to 200 $\mathrm{keV}$. The ASM observations reveal that the soft X-ray lightcurve shows a similar outburst to that detected by INTEGRAL, however the peak of the soft X-ray lightcurve either lags, or is preceded by, the hard X-ray ( $>20 \mathrm{keV}$ ) outburst by $\sim 2$ days. This resembles the behavior of X-ray novae like XN 1124-683, hence it further suggests a LMXB nature for IGR J17098-3628. While the quality of the ASM data prevents us from drawing any definite conclusions, these discoveries are important clues that, coupled with future observations, will help to resolve the as yet unknown nature of IGR J17098-3628.
\end{abstract}

7th INTEGRAL Workshop

September 8-11 2008

Copenhagen, Denmark

\footnotetext{
* Speaker.
} 


\section{Introduction}

INTEGRAL has detected roughly 500 sources at energies $\geq 20 \mathrm{keV}$. Among them, X-ray binary systems were identifi ed for $32 \%$ of the times, and another $26 \%$ remained unidentifi ed. As one of the unidentifi ed sources, IGR J17098-3628 was discovered [1] at the end of March 2005 by IBIS during the private Open Program observation dedicated to the deep view to the Galactic center.

The source got a peak flux at $\sim 60$ and $95 \mathrm{mCrab}$ for $18-45$ and $45-80 \mathrm{keV}$, respectively. The 20-60 keV flux evolved from $\sim 50$ mCrab at 2005 March 26 00:00, to $~ 9$ mCrabs at April 3 23:30 (UTC) [2]. The preliminary spectral analysis [3] showed the source spectrum became quite soft along this evolution. Following INTEGRAL, IGR J17098-3628 was observed by RXTE on March 29 of 2005 , and was detected at $80 \mathrm{mCrab}$ in the 3-20 keV band, with a hard power-law tail of a spectral index $\sim 2.5$. Then the source was assumed a Black Hole Candidate (BHC) and X-ray Nova [4]. The column density was found to be less than $1 \times 10^{22}$ atoms $/ \mathrm{cm}^{2}$.

The most recent optical and infrared observations, made with the $6.5 \mathrm{~m}$ Magellan-Baade telescope, revealed that 2MASS J17094612-3627573 [5] is in fact composed of several sources [6]. Furthermore, VLA observations of IGR J17098-3628 were made on March 31, April 5, and May 4, 2005 at $4.86 \mathrm{GHz}$ [7], which led to the discovery of a radio transient at 0.8 arcseconds from the Swift XRT position [8], and hence regarded as the possible radio counterpart. Finally on July 9, Swift/XRT observed IGR J17098-3628 again and found that the spectrum was fi tted by an absorbed disk blackbody model. The column density obtained was $(0.89 \pm 0.02) \times 10^{22}$ atoms $\mathrm{cm}^{-2}$ [9]. In this poster, we report the analysis of all available observations on IGR J17098-3628 carried out by IBIS/ISGRI and JEMX onboard INTEGRAL, and the All Sky Monitor (ASM) onboard RXTE. This allows us to trace the source behavior in X-rays back to 1996 with ASM and back to 2002 with INTEGRAL. We put the outburst in 2005 in this context.

\section{Time variability monitored by ASM}

IGR J17098-3628 has been monitored by ASM as one of the most active sources ever seen since 2002. As shown in Figure 1, the $1.5-12 \mathrm{keV}$ lightcurves, retrieved in a time period from January 1996 to May 2008, show the dwell and monthly averaged lightcurves. The individual bursts-like events existing in the dwell lightcurve are fake due to the contamination from the neighboring source, GX349+2. But in general, GX349+2 is stable in the $1.5-12 \mathrm{keV}$ band observed by ASM (see Figure 1, the bottom panel). In short, the analysis software has incorrectly attributed the flux from GX349+2 to IGR J17098-3628 only on those relatively few occasions. The sources are well separated by the INTEGRAL instruments. In the monthly averaged lightcurve there are several well established outbursts with a duration of several months. The fi rst one occurs around MJD 52800 (June, 2003), with averaged peak flux reaching $40 \mathrm{mCrab}$ level. We will see later that, this outburst came from the IGR J17091-3624 [4], and the ones thereafter observed by INTEGRAL were from IGR J17098-3628. IGR J17091-3624 seems to be in quiet most of time since MJD 52800 [4]. A sequence of outbursts from IGR J17098-3628 have happened since MJD 53455 (March, 2005), and the 1.5-12 keV flux has kept staying at the flux level of $\sim 50 \mathrm{mCrab}$ over years. These results, together with the INTEGRAL results (see below), indicate that IGR J17098-3628 


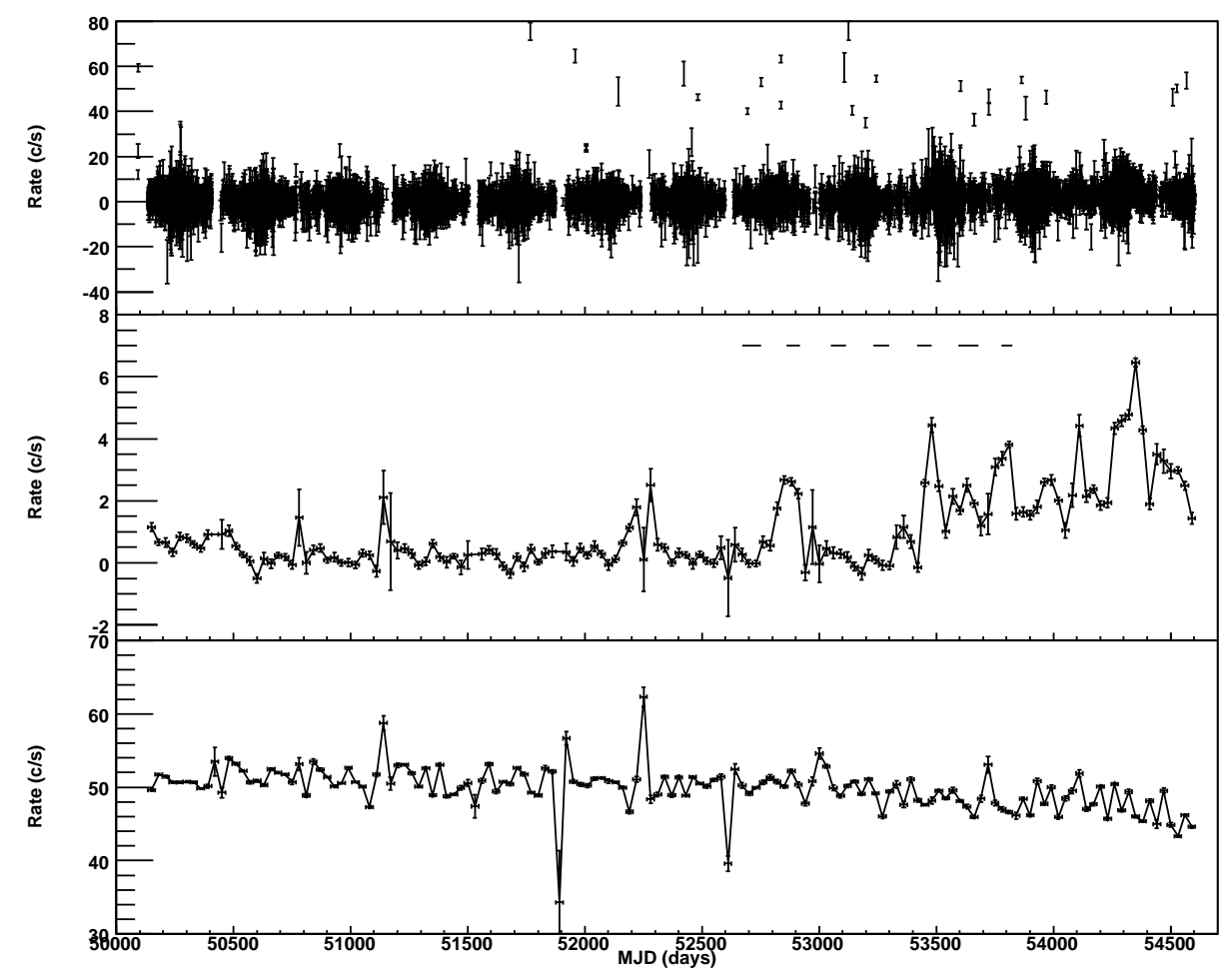

Figure 1: ASM light curves (1.5-12 keV) of IGR J17098-3628 with each bin representing a dwell (top) or average over month (middle) timescales between 1996 and 2008. In the middle panel, the available INTEGRAL observations are shown by the bars. ASM light curve (1.5-12 keV) of GX349+2 is presented in the bottom panel, with each bin representing average over month timescales between 1996 and 2008. In general, GX349+2 is stable in the $1.5-12 \mathrm{keV}$ band observed by ASM.

has been in active phase during the past several years.

\section{Hard X-ray properties revealed by INTEGRAL}

\subsection{Observations and data analysis}

The available INTEGRAL observations comprise about 2400 science windows, adding to a total exposure of $3700 \mathrm{ks}$ for the IBIS/ISGRI instrument. The data analysis is performed by using the INTEGRAL OSA version 6.0. All the sources within the FoV that are brighter than or comparable to IGR J17098-3628 are taken into account in extracting the source spectrum. An additional $2 \%$ systematic error are added to the spectra because of calibration uncertainties. The spectra are fi tted with XSPEC v12.3.1 and the model parameters are estimated with 90\% confi dence level.

\subsection{INTEGRAL results}

\subsubsection{Sky maps}

IGR J17098-3628 was detected by ISGRI at energies $\geq 20 \mathrm{keV}$ during March 24-28, 2005 when the source had an outburst in hard X-rays. The detection signifi cances were $73.4 \sigma$ in the 
$20-40 \mathrm{keV}$ band, $38 \sigma$ in the $40-60 \mathrm{keV}$ band, $21 \sigma$ in the $60-100 \mathrm{keV}$ band, and $8 \sigma$ in the $100-$ $200 \mathrm{keV}$ band. The source was still visible during its decay in the following one month, with the detections signifi cances dropping to $16.9 \sigma$ in $20-40 \mathrm{keV}$. For all other INTEGRAL observations, only marginal detections or upper limits were obtained at energies $\geq 20 \mathrm{keV}$, e.g., for the time period 2005 Apr. 2-21, the source was detected only in 20-40 keV with signifi cance $\sim 8 \sigma$ level. Since JEMX overlaps with ASM in most of its working energies, the detections at $\leq 15 \mathrm{keV}$ follow in general the same trend indicated by the ASM light curves.

\subsubsection{Light curves}

A comparison between the JEMX and ASM lightcurves at 3-12 keV shows the trends consistent with each other. We fi nd that the soft X-ray (3-12 keV) lightcurve shows a similar outburst to that detected by JEMX, however the peak of the soft X-ray (ASM 1.5-12 keV) lightcurve lags the hard X-rays (IBIS/ISGRI 20-80 keV) by $\sim 2$ days (Figure 2). We note, however, that no hard X-ray data cover the peak outburst observed in the ASM data. If there is a hard X-ray peak that coincides with the soft X-ray peak, then, since there is no evidence for two peaks in the ASM data, the earlier hard X-ray peak may well be interpreted as a precursor event to a wider band concurrent X-ray outburst rather than evidence for a lag between the hard and soft X-ray bands. Figure 3 shows a comparison of the flux variability in the time zones until $2006 \mathrm{Feb} 16$, as obtained with ASM in $1.5-3 \mathrm{keV}$, JEMX in 3-6, 6-10, 10-15 keV, and ISGRI in 20-40 keV, and 40-60 keV bands.

\subsubsection{Spectra}

Since the lightcurves show quite different behavior between the higher and lower energy bands, we investigate the spectrum at the different periods of the outburst and subdivide the time zone where the outburst was recorded by INTEGRAL into three smaller time periods: March 23 03:13 to 25 02:35(the rising phase), 25 23:03 to 27 00:51 (the peaking phase) and 27 00:53 to 28 11:15 (UTC) (the decaying phase). Due to the relatively small FoV of the JEMX, its data are only available during the decaying phase, which is then combined with the corresponding ISGRI data for a broader spectral fi tting. We fi nd that the relatively poor statistics of the data from the rising of the hard X-ray outburst prevents from discriminating the models; the power-law with cutoff model might be the best choice when the outburst reached the maximum; and the power-law model shows a good fi t to the data from the decay of the outburst onwards. From the fit of a simple power law model, the spectral index changes from $\sim 1.7$ when rising to roughly 2.6 when decaying. That the different episodes of the outburst have to be described by different models might suggest rather strong time evolution. By taking the distance of $10.5 \mathrm{kpc}$ as estimated by Grebenev et al.(2007), the source had a luminosity of $\sim 9.8 \times 10^{36} \mathrm{erg} \mathrm{s}^{-1}$ averaged over the whole outburst in the 20-200 $\mathrm{keV}$. The luminosities in individual phase are $5.3 \times 10^{36} \mathrm{erg} \mathrm{s}^{-1}$ (the rising), $1.6 \times 10^{37} \mathrm{erg} \mathrm{s}^{-1}$ (the peaking) and $8.9 \times 10^{36} \mathrm{erg} \mathrm{s}^{-1}$ (the decaying).

\section{Discussion and summary}

IGR J17098-3628 may have stepped into an active phase in the beginning of 2005. INTEGRAL detected a days-long outburst extending up to energies of $\sim 200 \mathrm{keV}$. A similar outburst is 


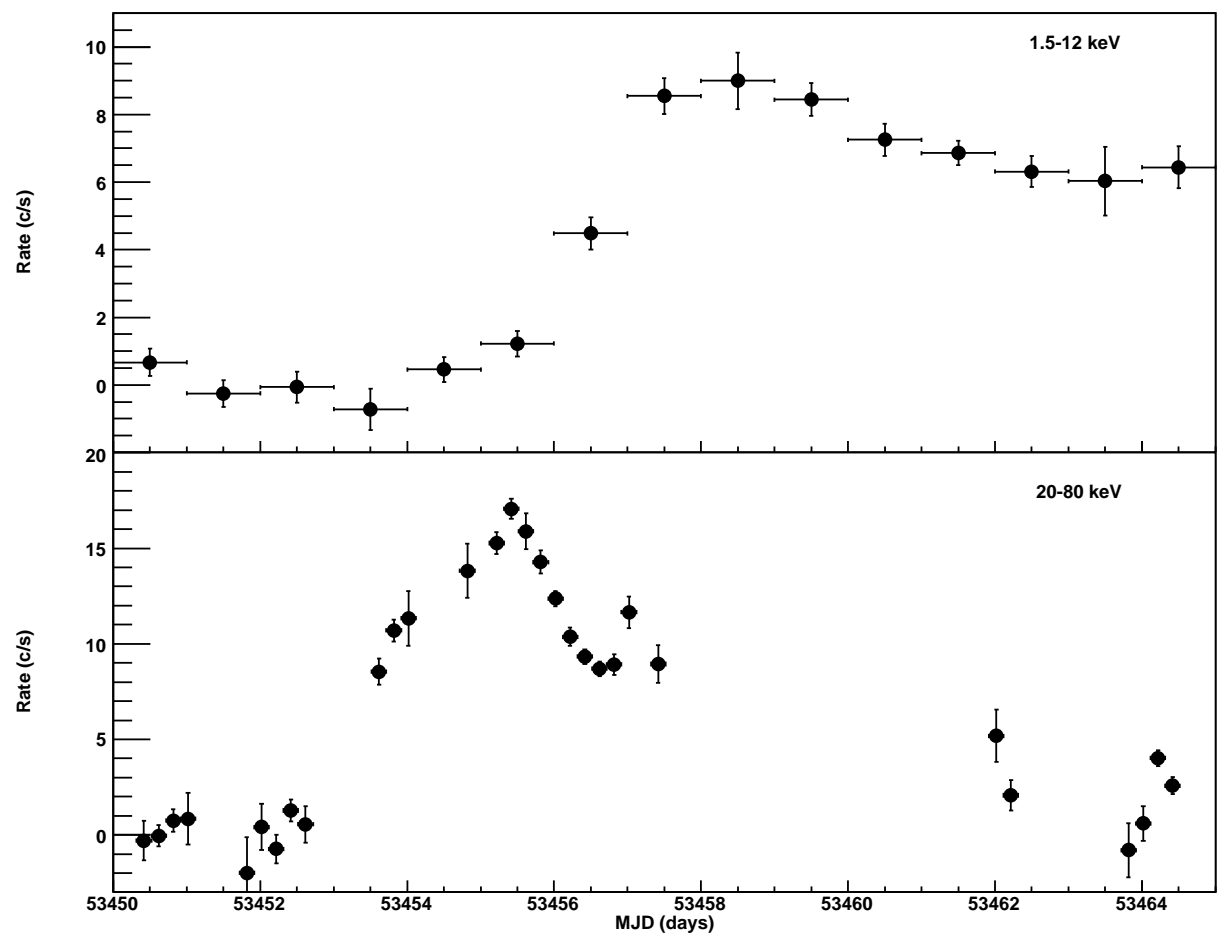

Figure 2: Lightcurves of IGR J17098-3628 covering the March 2005 days-duration outburst. Upper panel: 1.5-12 keV ASM lightcurve (1-day binsize). Lower Panel: 20-80 keV INTEGRAL/ISGRI lightcurve (0.2day bin size).

detected in the soft X-ray (1.5-12 keV) band, however the onset of the outburst lags the hard X-ray outburst by $\sim 2$ days, in a fashion reminiscent of X-ray novae like XN 1124-683 [10] The spectral analysis of the initial stages of the outburst detected by INTEGRAL supports the association with $\mathrm{X}$-ray novae [4], the cool disk temperature and small inner radius of the accretion disk both suggest that IGR J17098-3628 is a newly discovered black hole system. Unfortunately, the lack of any detailed X-ray spectra of the IGR J17098-3628 outburst prevents us from drawing any conclusions on the X-ray nova mechanism for IGR J17098-3628. That the three episodes of the hard X-ray outburst observed by INTEGRAL have to be described by different models might suggest rather strong time evolution, i.e., the spectral index changed from $\sim 1.7$ when rising to roughly 2.6 when decaying suggesting the cooling of the source.

Although the time variability of X-ray novae is not well understood theoretically, observations of a variety of X-ray novae not only show that the hard X-ray flux often rises and peaks earlier than the outburst in soft X-rays, but can also show precursor events at hard X-rays several days prior to the main outburst [10]. One potential mechanism that may reproduce the observed lag is for an instability in the disk to trigger the formation of a temporary jet, which produces hard X-rays via inverse comptonization, in advance of any change in the flux of soft X-rays from the disk.

A more straightforward scenario in understanding this different time behaviour between the hard and the soft X-rays may lie in the tie-up with the structure of the accretion flow. The precursor event can be explained as the transition from the "hard" state to "soft" state in the BHC system. 

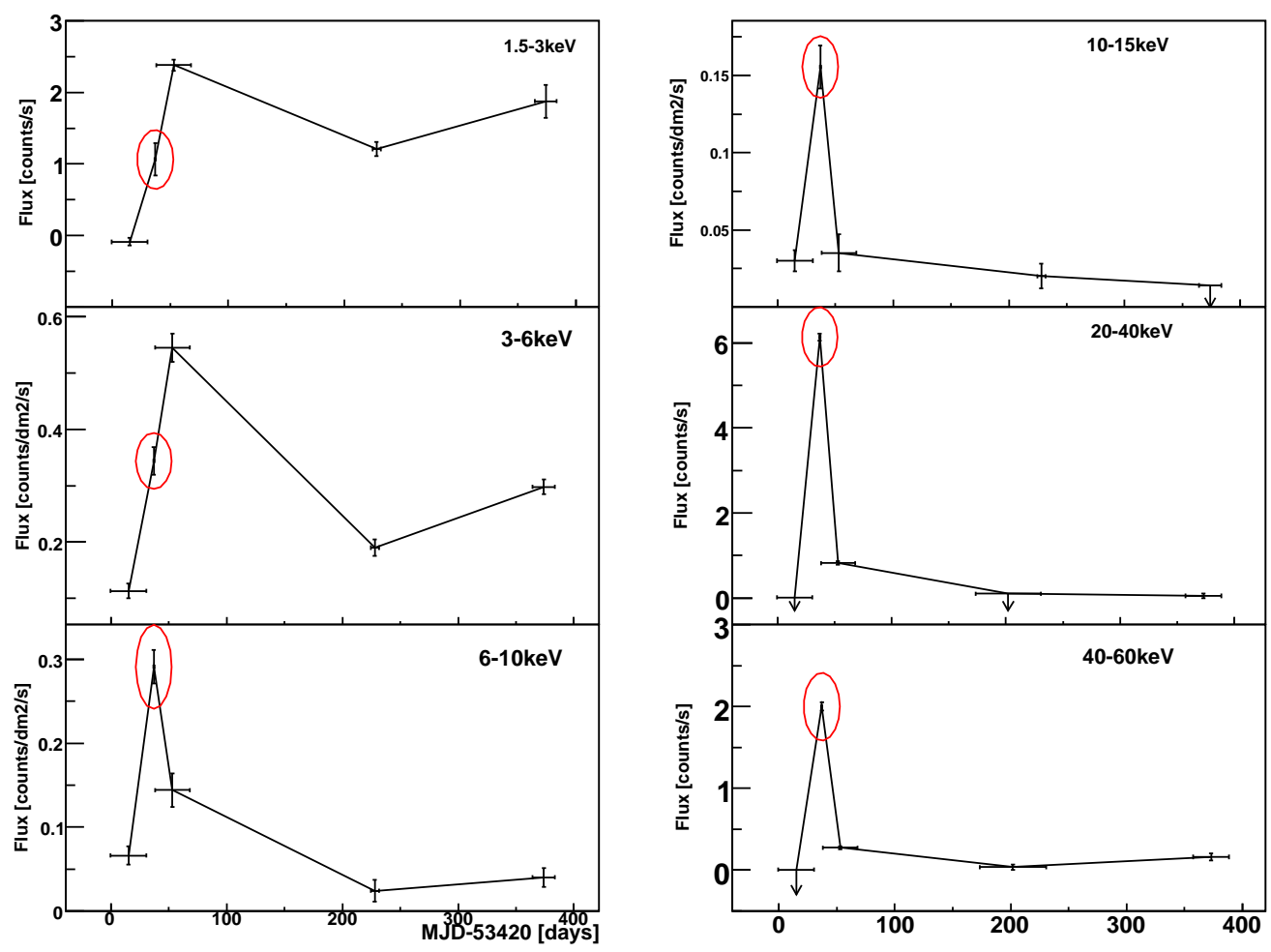

Figure 3: Lightcurves from ASM (1.5-3 keV), JEMX (3-6keV, 6-10, 10-15 keV) and ISGRI (20-40 keV, $40-60 \mathrm{keV})$. The hard $\mathrm{X}$-ray outburst is marked by the red circle.

The accretion time in the inner parts of the disk is much smaller than the duration of X-ray nova's outburst therefore changes in the spectral state of these sources are connected with general changes in structure of the accretion flow rather than in changes in number of soft seed photons for Comptonization. Observations show that all BHC systems have the hard Comptonized spectrum at low accretion rates and the soft disc blackbody spectrum at high accretion rates. This may be connected with approaching of the inner radius of a cold accretion disk to the radius of marginally stable orbit when the accretion rate increases. The observed lag between outbursts in hard X-rays and soft $\mathrm{X}$-ray may be directly connected with this observed dependence: the accretion rate is small at the initial stage of the outburst thus the spectrum is hard and the outburst is observed in hard X-rays, but later the accretion rate rises and the spectrum becomes soft thus the outburst is observed in soft $\mathrm{X}$-rays. More details can be found in [11].

In summary, although no clear statement can be made regarding their nature, the discovery the lag/precursor event revealed by the concurrent RXTE ASM and INTEGRAL observations in 2005, is important piece of the IGR J17098-3628 puzzle that will help to resolve the as yet unknown nature of this perplexing source.

\section{References}

[1] Grebenev, S.A., Molkov, S.V., \& Sunyaev R.A. 2005, ATel, 444. New X-ray transient IGR J1709.8-3628 discovered with INTEGRAL, ATel 444 (2005) 
[2] Mowlavi, N., Kuulkers, E., Rodriguez, J., et al. 2005, ATel, 453. INTEGRAL monitoring of IGR J17098-3628, ATel 453 (2005)

[3] Grebenev, S.A., Molkov, S.V., Revnivtsev, M.G., \& Sunyaev, R.A. 2005, ATel, 447 Spectral evolution of IGR J17098-3628 observed by INTEGRAL and RXTE, ATel 447 (2005)

[4] Grebenev, S. A., Molcov, S. V., Revnivtsev, M.G., \& Sunyaev, R.A. 2007, Proceedings of the 6th INTEGRAL Workshop 'The Obscured Universe', ESA SP-622 373 (2007)

[5] A.K.H. Kong. 2005, ATel, 477 Possible infrared/optical counterpart of IGR J17098-3628, ATel 477 (2005)

[6] Steeghs, D., Torres, M.A.P., Jonker, P.G., et al. 2005, ATel, 478 Optical/NIR imaging of IGR J17098-3628 and IGR J16283-4838, ATel 478 (2005)

[7] Rupen, M.P., Mioduszewski, A.J., \& Dhawan, V. 2005, ATel, 490 Probable radio counterpart to IGR J17098-3628, ATel 490 (2005)

[8] Kennea, J.A., D. N. Burrows, J. A. Nousek et al. 2005, ATel, 476 Swift-XRT detection of IGR J17098-3626, ATel 476 (2005)

[9] Kennea, J.A., Capitanio, F. 2007, ATel, 1140 Swift/XRT Observations of IGR J17091-3624 and IGR J17098-3628, ATel 1140 (2007)

[10] Chen, W. , Shrader, C.R., \& Mario L., The Properties of X-Ray and Optical Light Curves of X-Ray Novae, ApJ 491312 (1997)

[11] Yu-Peng Chen, Shu Zhang, Nick Schurch et al., IGR J17098-3628 Observed by INTEGRAL and RXTE/ASM, PASJ 601173 (2008) 